\title{
Potential benefits of oral administration of AMORPHOPHALLUS KONJAC glycosylceramides on skin health - a randomized clinical study
}

\author{
Sudeep Heggar Venkataramana*, Naveen Puttaswamy and Shyamprasad Kodimule
}

\begin{abstract}
Background: Ceramides play a fundamental role in maintaining the skin health as a function of improved barrier permeability. Reduced ceramide content results in skin dryness and wrinkledness. Intake of dietary ceramides potentially compensates the skin ceramide content. In the present study we have assessed the skin health benefits of oral supplementation of a hydroalcoholic extract from Amorphophallus konjac tubers standardized to 5\% glycosylceramides, in a placebo-controlled clinical trial.

Methods: Fifty-one healthy human volunteers (aged 18-60 years) were supplemented with $100 \mathrm{mg} /$ day of either a placebo or A. konjac extract capsules (5 mg glycosylceramides) for 6-weeks. The skin parameters were evaluated through dermatological diagnosis. Subject perceived efficacy of the product was further evaluated by a selfassessment questionnaire.

Results: Oral intake of $A$. konjac extract significantly decreased the skin dryness, hyperpigmentation, redness, itching and oilyness $(p<0.05)$. The improvement in skin health following intake of $A$. konjac extract was observed to be time-dependent from the start. Further, A. konjac extract was well-tolerated throughout the study, as no adverse events or toxic changes were recorded.
\end{abstract}

Conclusion: The study demonstrates the skincare properties of orally ingested glycosyl ceramides from konjac tubers.

Trial registration: CTRI/2018/12/016661 dated 13/12/2018 retrospectively registered, http://ctri.nic.in/Clinicaltrials/ showallp.php?mid1=19851\&EncHid=\&userName=SkinCera

Keywords: Phytoceramides, Skin barrier function, Human clinical

\section{Background}

Ceramides are a variety of sphingolipids, present in the skin keeping it moist and healthy [1]. Human epidermis is composed of the stratum basale, stratum spinosum, granulosa, and stratum corneum from the inner layer towards outside. In the stratum basale, phospholipids and cholesterol are the main components while granulosa majorly contains glycosylceramides. Ceramide in the stratum corneum plays a key role in barrier functions

\footnotetext{
* Correspondence: sudeepkashyap.82@vidyaherbs.com; sudeepkashyap.82@gmail.com

Research Scientist, No. 14A, KIADB, R \& D Center for Excellence, Vidya Herbs (P) Ltd., Jigani Industrial Area, Bangalore, Anekal Taluk 560 105, India
}

such as moisture retention, maintenance of skin architecture, prevention of invasion of foreign bodies [2, 3].

During the recent past, studies have demonstrated the beneficial effects of orally supplemented ceramides against dry skin, skin aspect, and associated discomforts. Animal studies have been conducted to describe the oral bioavailability of ceramide. Dietary glycosylceramides are metabolized in rat small intestine and found in portal blood after hydrolysis by ceramidases in the gastrointestinal tract [4]. Nevertheless, a large proportion of ingested sphingolipids are excreted in the feces, animal studies suggest that after oral intake radiolabeled ceramides are metabolized, absorbed and distributed to many tissues, including the 


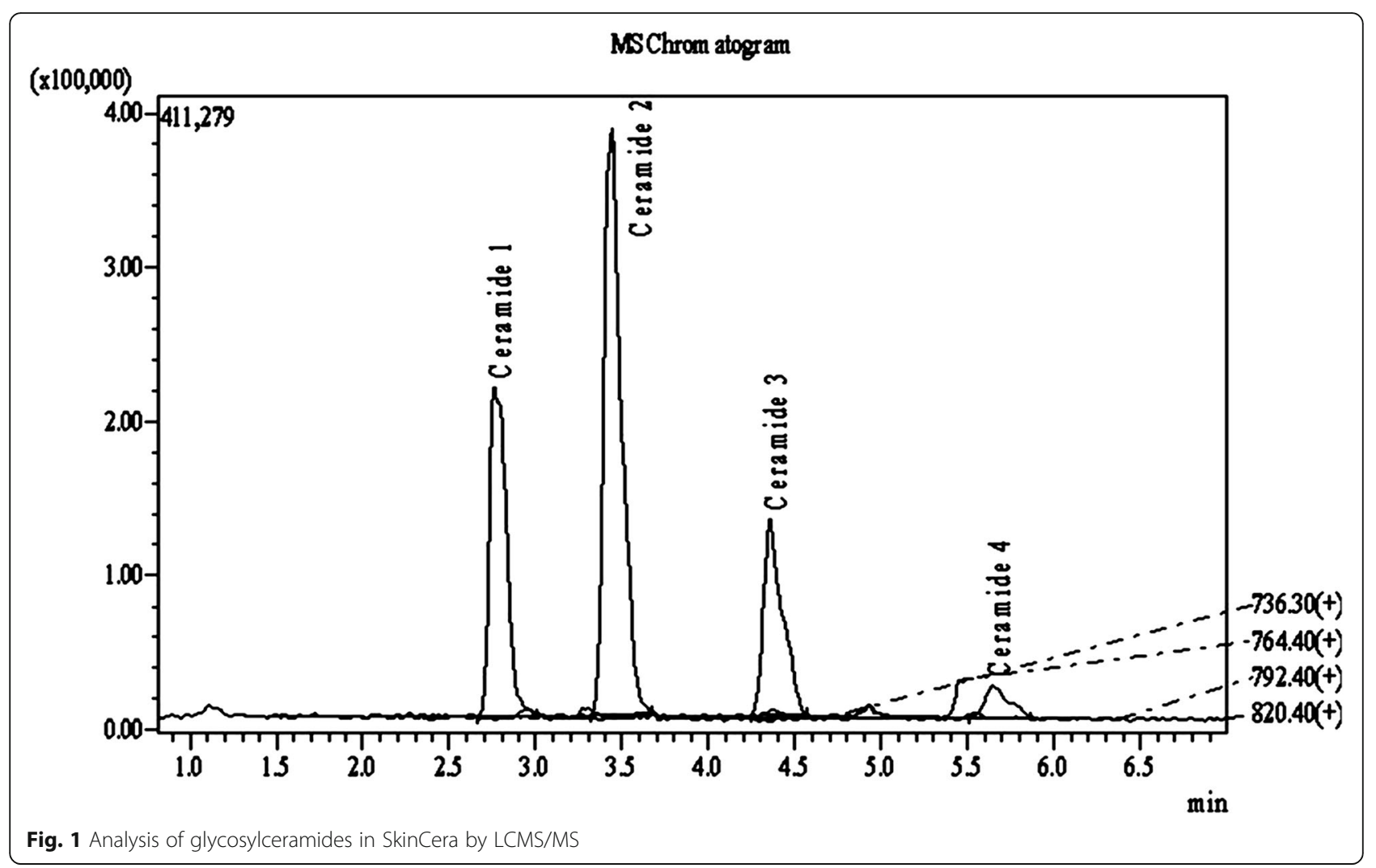

skin [5]. Ueda et al., showed that orally administrated radiolabeled D2-sphingosine is transferred to the skin, from dermis to epidermis in an unchanged structural form, and further generates radiolabeled glucosylceramides and ceramides by in vivo biosynthesis in mice [6]. The orally ingested sphingolipids in human beings could be hypothesized to follow a similar track of metabolism as described in animal studies. Not all the ingested sphingolipids are hydrolyzed and absorbed. However, some components of sphingolipids (sphingosine) reach the systemic circulation following transport through the mucosa. The dietary ceramides may activate the ceramide synthesis in the skin improving the barrier function, rather than direct reutilization reaching the skin [7]. In addition, previous studies have demonstrated that oral supplementation with ceramides may be beneficial for skin permeability barrier homeostasis and parameters such as hydration and/or barrier function, elasticity, and recovery after induced disruption of barrier dysfunction [8-10].

Loss of ceramides causes dry skin and dermatitis that subsequently leads to the appearance of wrinkle in the skin [11]. Supplementation of ceramides thus is essential for maintaining skin barrier permeability and hydration. Nevertheless, synthetic ceramides are used in general as cosmetics; natural ceramides have drawn much attention during the recent past due to the safety [12]. Plant derived ceramides are chemically identical to those found in our skin. Currently there are several types of ceramides available in the market commercially derived from plant sources such as rice, wheat, soy, and spinach. Glycosylceramides have been reported to improve skin barrier function in hairless mice by intake of extract of food materials containing glycosylceramide. Oral intake of glycosylceramide reduces transepidermal water loss in normal adult or in atopic dermatitis patients [13].

Amorphophallus konjac (Family: Araceae) is a perennial plant commonly known as konjak or konnyaku. Konjac is a traditional food ingredient and medicine used in China, Japan and South East Asia. konjac is used in the Chinese folk medicine as a tumour suppressor, detoxification and phlegm liquefaction. A. konjac is a rich source of glycosyl ceramides and glucomannan.

Table 1 Quantitative formula of treatments (per capsule)

\begin{tabular}{lll}
\hline Composition & SkinCera $(\mathrm{mg})$ & Placebo $(\mathrm{mg})$ \\
\hline Maltodextrin & 70 & 120 \\
A. konjac extract & 50 & 0 \\
Dicalcium phosphate & 100 & 100 \\
Magnesium carbonate & 50 & 50 \\
Magnesium stereate & 5 & 5 \\
Silica micronized & 25 & 25 \\
Total weight & 300 & 300 \\
\hline
\end{tabular}




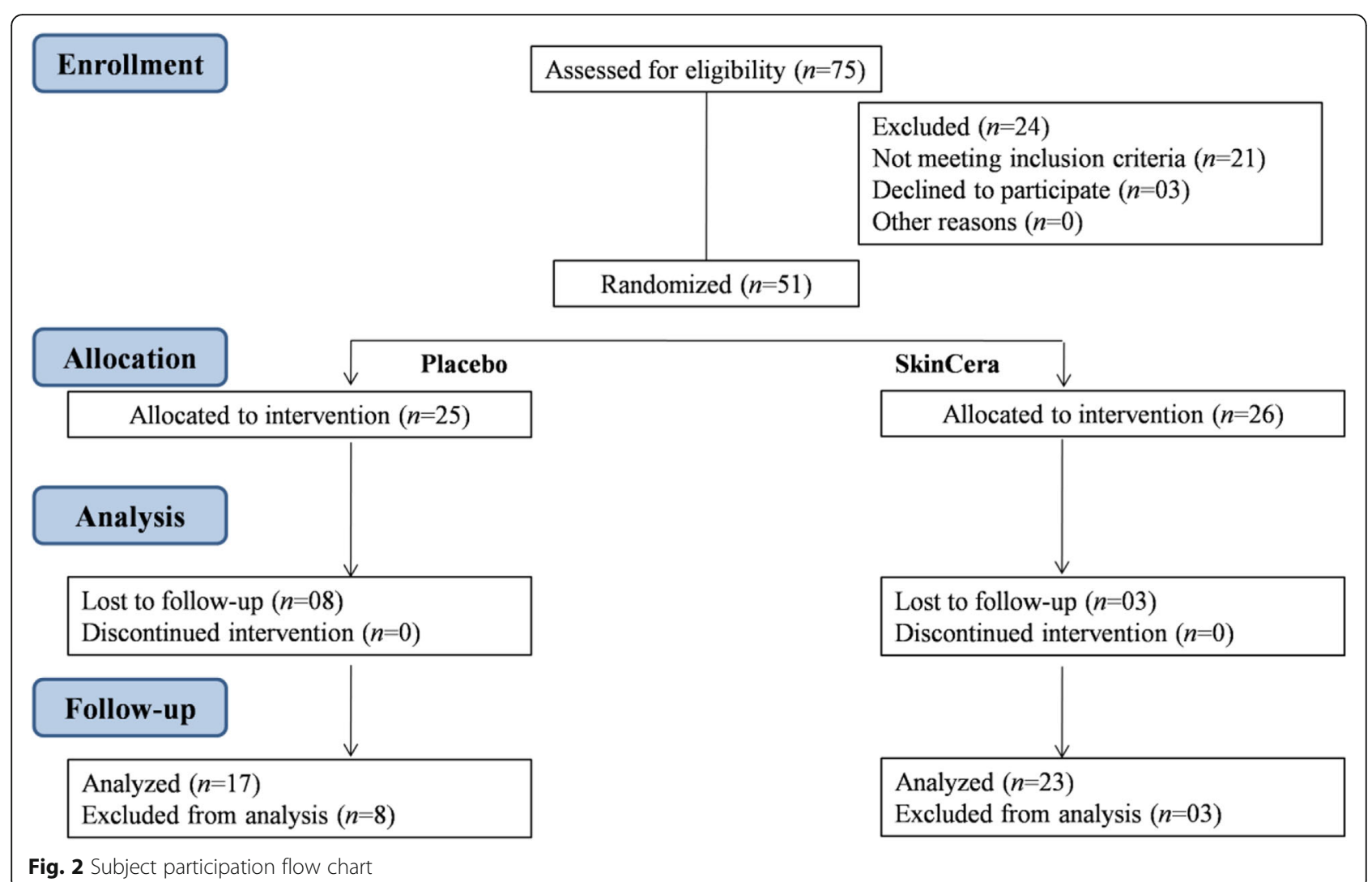

Konjac tuber-derived glycosylceramides have been commercially exploited as dietary supplements for dry skin [14]. Previous studies in mice have shown that konjac glycosylceramides are effective against trans-epidermal water loss [9]. Usuki et al. [14] have reported the efficacy of konjac ceramides on itch-causing neurite outgrowth in PC12 cells [15]. Based on the experimental and clinical evidences from previous studies we have evaluated the effects of orally ingested konjac extract containing glycosylceramides on skin care properties in a placebocontrolled, randomized, single-blind clinical trial with healthy human volunteers.

\section{Methods}

\section{Investigational product}

This study was conducted to investigate the efficacy of SkinCera $^{\mathrm{am}}$, a proprietary extract from the tubers of $A$. konjac. The formal identification of the plant material was done at Vidya Japan K.K., Minato-ku, Tokyo, Japan. SkinCera is a hydroalcoholic extract standardized to $5 \%$ of glycosyl ceramides.

\section{Trial design}

This was a monocentric single-blinded, placebo-controlled, randomized study performed on 50 healthy human volunteers. The study was conducted in compliance with the protocol, International Conference on Harmonization
(ICH) Good Clinical Practice (GCP) Guidelines, including ICH E6, and applicable local regulatory requirements and laws. This clinical trial adheres to the CONSORT guidelines. The information on the nature, purpose, and risks of the study were provided to each subject or subject's legally authorized representative before their participation. Written informed consent for participation and publication of the data was obtained prior to the subject entering the study (before initiation of protocol-specified procedures). The subjects were randomized to two intervention groups: placebo and $A$. konjac extract (capsule form, $100 \mathrm{mg} /$ day).

\section{Ethics, consent and permissions}

The study protocol and informed consent form were approved by institutional ethics committee (SKAMC/96/ 2017-18, Sri Kalabyraveshwara Swamy Ayurvedic Medical College, Hospital and Research Center, India). This clinical study was registered in Clinical Trials Registry - India

Table 2 Baseline demographics of subjects

\begin{tabular}{lll}
\hline & SkinCera & Placebo \\
\hline Number of subjects & 26 & 25 \\
Age (years) & $28.08 \pm 7.3$ & $32.56 \pm 5.3$ \\
Height & $163.2 \pm 10.3$ & $165.8 \pm 7.27$ \\
Weight & $62.4 \pm 14.63$ & $65.9 \pm 6.82$ \\
\hline
\end{tabular}

The data were statistically analyzed at $p<0.05$ and represented as mean \pm SD 
(CTRI/2018/12/016661). Informed consent and consent to publish were obtained prior to subject randomization. Subjects meeting all inclusion and no exclusion criteria signed a written informed consent and enrolled in the study.

\section{Participants}

\section{Eligibility criteria}

Healthy adult male and female volunteers aged 18-60 years presenting symptoms such as skin dryness, roughness, itching, redness, hyperpigmentation, blackheads and whiteheads were recruited. Subjects with chronic medical disorders were excluded from the study. Other exclusion criteria were pregnant or lactating women, smokers and alcoholics, subjects undergoing other cosmetic treatments. Further, subjects unwilling/unable to comply with the protocol requirements were excluded from the study.

\section{Study site}

The study was performed at Sri Kalabyraveshwara Swamy Ayurvedic Medical College, Hospital and Research Center, Bangalore, India.

\section{Interventions}

A standardized extract from $A$. konjac tubers was prepared according to a proprietary manufacturing process using solvent based extraction strategy. The raw material was procured from Gunma prefecture, Japan and authenticated internally at Vidya Herbs Pvt. Ltd., Japan. A. konjac extract is standardized to $\geq 5 \%$ glycosylceramides (ceramide 1-4, Fig. 1) by LCMS/MS analysis. Further details on the constituents of $A$. konjac extract are provided in supplementary file (Additional file 3).
During the 6-week treatment period, the daily oral intake was two capsules containing either placebo (maltodextrin) or $A$. konjac extract $(=100 \mathrm{mg} /$ day). Indeed, SkinCera group received $5 \mathrm{mg} /$ day of glycosylceramides. All capsules were of the same appearance, color and odor. Table 1 shows the composition of capsules. The subjects had to record their daily food consumption during the intervention period to ensure that there was no change in the dietary habits throughout study period.

\section{Outcomes}

The primary end point was to assess the effect of $A$. konjac extract consumption on skin health. The efficacy evaluation was performed after a regular interval of 3 weeks of ingestion: Visit 1 (day 1), visit 2 (third week of treatment) and visit 3 (after 6 weeks of treatment). The skin parameters included in the study were evaluated as overall symptoms through dermatological diagnosis and inquiry. Diagnosis score is usually a number that conveys the response of a subject to the treatment (Additional file 2). With reference to the previously validated subjective scoring scales, the diagnosis score test was developed by the investigator before the conduct of study [16, 17]. In each evaluation, anchor points were prepared and evaluated by the investigator. A total of eight skin parameters were considered for the study and the subjects were evaluated by the investigator at every scheduled visit. The safety analysis was summarized for vital signs and adverse event monitoring.

The subject perception of the product efficacy was evaluated by a validated self-assessment questionnaire at follow-up visit.

Table 3 Dermatological diagnosis before and after 6-week treatment with SkinCera

\begin{tabular}{|c|c|c|c|c|c|c|c|c|}
\hline \multirow[b]{2}{*}{ Parameter } & \multicolumn{4}{|l|}{ SkinCera group } & \multicolumn{4}{|l|}{ Placebo group } \\
\hline & $\begin{array}{l}\text { Number of subjects } \\
\text { with symptoms }\end{array}$ & $\begin{array}{l}\text { Before } \\
\text { treatment }\end{array}$ & After 3 weeks & After 6 weeks & $\begin{array}{l}\text { Number of subjects } \\
\text { with symptoms }\end{array}$ & $\begin{array}{l}\text { Before } \\
\text { treatment }\end{array}$ & $\begin{array}{l}\text { After } 3 \\
\text { weeks }\end{array}$ & $\begin{array}{l}\text { After } 6 \\
\text { weeks }\end{array}$ \\
\hline Dryness & 8 & $1.88 \pm 0.99$ & $0.5 \pm 0.75^{* *}$ & $0.63 \pm 0.51^{* *}$ & 12 & $1.33 \pm 0.49$ & $1.58 \pm 0.51$ & $\begin{array}{l}1.67 \pm \\
0.65\end{array}$ \\
\hline $\begin{array}{l}\text { Whiteheads/ } \\
\text { blackheads }\end{array}$ & 10 & $1.56 \pm 0.72$ & $1.0 \pm 0.86$ & $1.11 \pm 0.78$ & 11 & $1.91 \pm 0.83$ & $2.46 \pm 0.52$ & $\begin{array}{l}2.55 \pm \\
0.69\end{array}$ \\
\hline Hyperpigmentation & 21 & $1.47 \pm 0.77$ & $1.05 \pm 0.52$ & $1.05 \pm 0.40^{*}$ & 11 & $2.46 \pm 0.52$ & $2.36 \pm 0.5$ & $\begin{array}{l}2.14 \pm \\
0.69\end{array}$ \\
\hline Redness & 10 & $1.7 \pm 0.67$ & $1.2 \pm 0.91$ & $0.6 \pm 0.84^{*}$ & 5 & $1.20 \pm 0.44$ & $1.8 \pm 0.44$ & LTF \\
\hline Lesions & 11 & $1.3 \pm 0.48$ & $1.1 \pm 0.87$ & $0.7 \pm 0.82$ & 5 & $1.4 \pm 0.54$ & $1.8 \pm 0.45$ & LTF \\
\hline Itching & 10 & $1.67 \pm 0.5$ & $0.88 \pm 0.78$ & $0.67 \pm 0.86^{*}$ & 3 & $1.33 \pm 0.57$ & $2.33 \pm 0.57$ & LTF \\
\hline Oilyness & 12 & $1.46 \pm 0.52$ & $0.90 \pm 0.83$ & $0.73 \pm 0.78^{*}$ & 9 & $1.11 \pm 0.33$ & $1.22 \pm 0.44$ & $\begin{array}{l}1.71 \pm \\
0.48^{*}\end{array}$ \\
\hline Roughness & 7 & $1.29 \pm 0.48$ & $0.71 \pm 0.75$ & $0.57 \pm 0.78$ & 11 & $1.91 \pm 0.83$ & $2.27 \pm 0.47$ & $\begin{array}{l}2.36 \pm \\
0.81\end{array}$ \\
\hline Overall & 26 & $1.53 \pm 0.66$ & $0.95 \pm 0.76^{* * *}$ & $0.79 \pm 0.71^{* * *}$ & 25 & $1.67 \pm 0.74$ & $1.98 \pm 0.63^{*}$ & $\begin{array}{l}2.10 \pm \\
0.75^{* *}\end{array}$ \\
\hline
\end{tabular}

The values are the mean in each group; analyzed by one-way ANOVA followed by Dunnet's t test. The data were statistically significant at * $p<0.05$, ** $p<0.01$, ${ }^{* * *} p<0.001$ within the group. LTF, Lost to follow-up

0 (no symptoms) - 1 (mild) - 2 (moderate) - 3 (severe) 


\section{Sample size}

The sample size calculation was based on the expected difference between mean scores of the two treatments considered to be medically relevant. Assuming a common standard deviation of 1.8 for the number of assessment parameters at the end of treatment, 20 per group would be enough to detect a difference of 1.63 in mean score difference between the two treatments with power of $80 \%$ and a 0.05 two-sided level of significance. Considering a dropout rate of $10 \%$ the sample size was finalized as 50 ( 25 per group). The details of the sample size calculation are presented as supplementary file (Additional file 4).

\section{Randomization and blinding}

The subjects were randomly assigned to the study groups in a 1:1 ratio to receive active treatment and placebo. Block randomization was used to assign the subjects to treatment groups. Each randomized subject received a xx-digit randomization number. Randomized subjects who terminated their study participation for any reason, regardless of whether the IP was taken or not, retained their randomization number. Subjects were kept blind to treatment group assignment.

\section{Statistical analysis}

The primary analysis was based on the full analysis set which included all randomized subjects and followed the intent-to-treat principle. Statistical analysis was performed using SPSS software version 16.0. All statistical significance tests were 2-sided and performed at the 5\% significance level. Data were analyzed by one-way ANOVA followed by Dunnet test.

\section{Results}

Subject enrollment and baseline data

A total of 75 subjects were screened from single study center and 51 volunteers were enrolled into the study. Out of these 51 subjects, 40 subjects completed the study and 11 were withdrawn from the study due to lost to follow-up/failed to return (Fig. 2). Subject baseline demographic data are presented in Table 2. Further details are provided in Additional file 1.

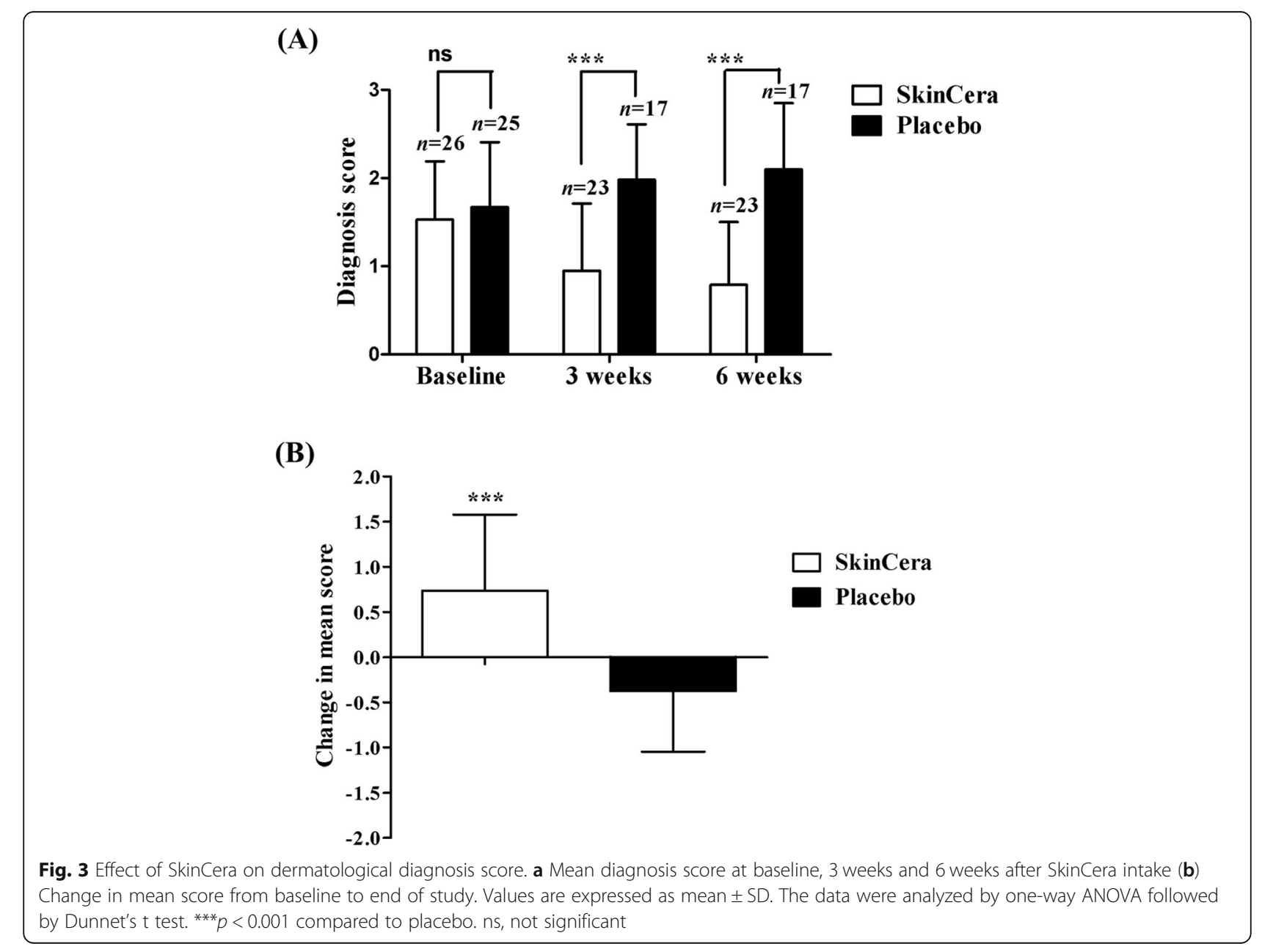




\section{Dermatological diagnosis}

The mean dermatological diagnosis score, before and after ingestion of $A$. konjac extract is presented in Table 3. In $A$. konjac extract treated group, the skin dryness was evident among 8 subjects with a mean severity symptom score of 1.88 at the screening visit (before treatment). The subjects showed significant improvement during the study having a mean score of 0.63 after 6 weeks treatment $(p<$ $0.01)$. Similarly, A. konjac extract treatment markedly decreased the mean score of hyperpigmentation from 1.58 at baseline to 1.053 after the 6 -week treatment $(p<0.05)$. Skin redness was observed initially among ten subjects in the $A$. konjac extract group with a mean score of 1.7. The subject response to skin redness, itching and oilyness following $A$. konjac extract treatment were significant at $p<0.05$ after 6 weeks. A. konjac extract treatment was appreciable for skin roughness though the data were not significant. Subject response in the A. konjac extract group for other skin parameters such as whiteheads/blackheads and lesions were moderate. It was further observed that the study parameters were aggravated among the subjects in the placebo group during the study. The overall mean diagnosis score decreased significantly $(p<0.001)$ after six-week oral ingestion of $A$. konjac extract (Fig. 3).

The percentage response of subjects following 6-week treatment with $100 \mathrm{mg} /$ day treatment with $A$. konjac extract, to individual skin parameters is presented in Fig. 4. The recovery percentage of subjects from skin dryness and roughness were highly appreciable among the various parameters assessed. Figure 5 shows the percentage improvement in the subject population following $A$. konjac extract treatment. A. konjac extract considerably

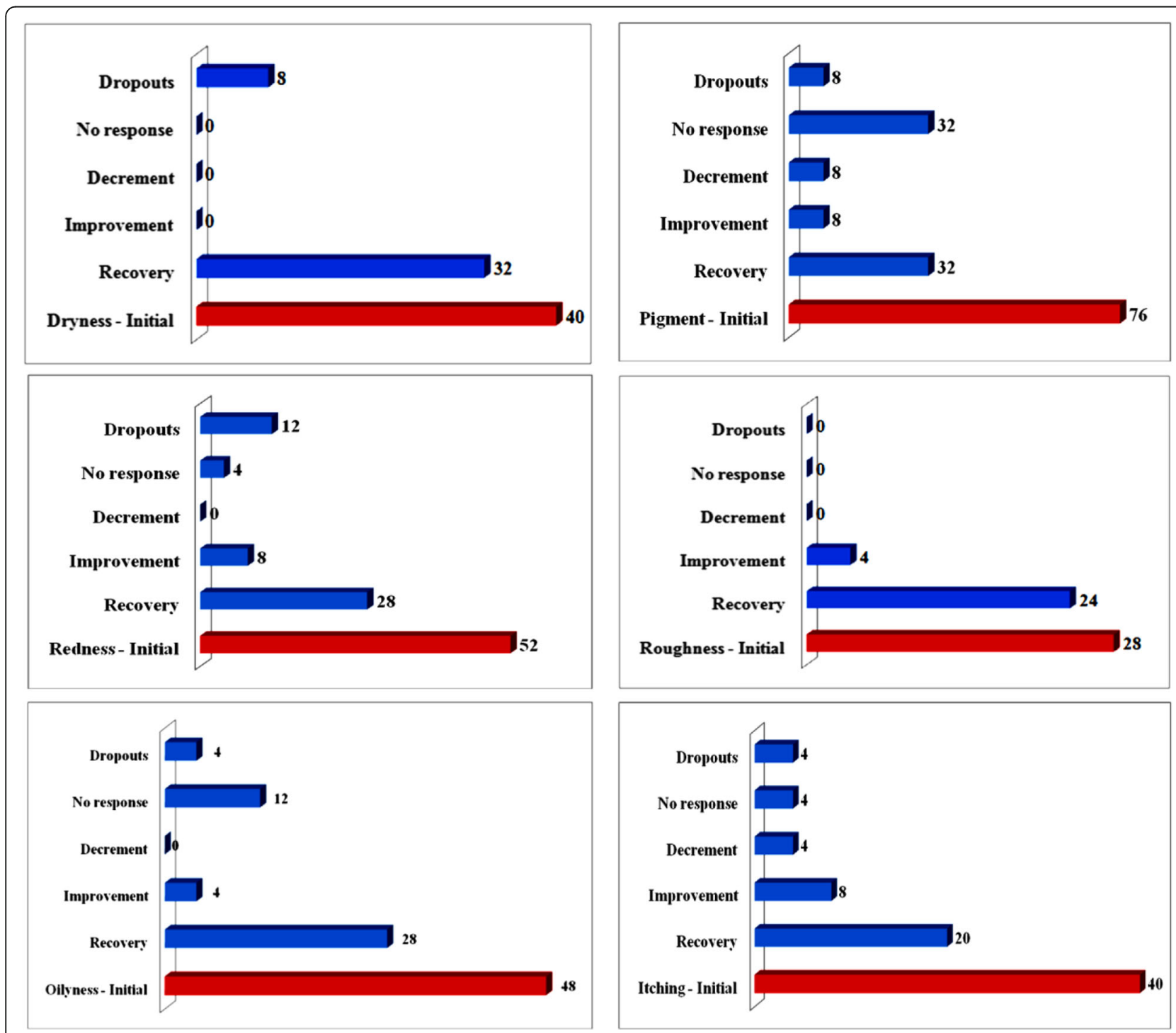

Fig. 4 Subject response (\%) to SkinCera treatment for individual skin parameters (at follow-up visit) 


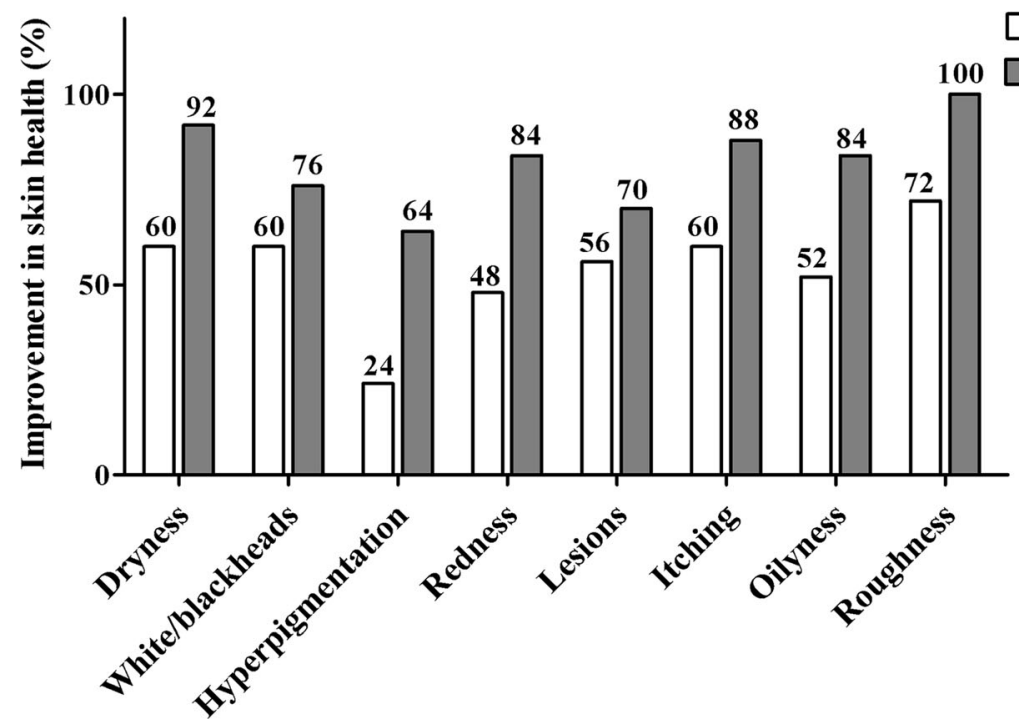

Fig. 5 Effect of SkinCera on the improvement in skin health from baseline to follow-up

alleviated the skin condition as evident from the increase in the percentage of subjects showing improvement for respective parameters.

\section{Adverse events}

A. konjac extract treatment did not induce any significant adverse or serious adverse effects throughout the study period as evident from the vital signs and health monitoring (Data not shown).

\section{Results of self-assessment questionnaire}

The subjects' perception as evaluated through selfassessment questionnaire was consistent with the results of the diagnosis. Results of the self-assessment questionnaire are presented in Table 4.

\section{Discussion}

Ceramides from edible plants have emerged as a safe and preferred alternative to animal ceramides for cosmetic applications [18]. Research on several plant-based ceramides are intended to supplement effective amount of the ceramide content to the skin which otherwise has reduced content of natural ceramides due to defective skin barrier function [9, 19]. Human clinical trials on ceramide-based products from various plant sources like wheat, rice, beet and konjac tubers have been documented to improve the skin barrier functions [8, 10, 20]. The clinical evidence on the efficacy of konjac glycosylceramides in other skincare properties apart from skin dryness is lacking. This clinical trial was undertaken to study the effect of supplementation of small quantity of glycosylceramides (5 mg/day) from konjac on overall skin health benefits in healthy human volunteers. A sixweek ingestion of $A$. konjac extract at $100 \mathrm{mg} /$ day (glycosylceramide, $5 \mathrm{mg} /$ day) significantly improved the skin parameters such as dryness, hyperpigmentation, itching and oilyness compared to placebo group. Interestingly, these beneficial effects were evident after 3-weeks of

Table 4 Results of self-assessment questionnaire after 6-week of supplementation with SkinCera

\begin{tabular}{lcc}
\hline Questions and self assessment & SkinCera & Placebo \\
\hline $\begin{array}{l}\text { How do you rate the efficacy of the product in skin dryness } \\
\text { improvement? }\end{array}$ & $0 \%$ & $65 \%$ \\
Insufficient & $30 \%$ & $30 \%$ \\
Sufficient & $35 \%$ & $5 \%$ \\
Fairly good & 35 & $0 \%$ \\
Excellent & \\
Do you think your skin texture is improved? & $30 \%$ \\
Yes & $75 \%$ & $60 \%$ \\
No & $20 \%$ & $10 \%$ \\
No opinion & $5 \%$ &
\end{tabular}

How do you evaluate the product efficacy concerning improvement in skin pigmentation?

$\begin{array}{lll}\text { Insufficient } & 20 \% & 75 \% \\ \text { Sufficient } & 30 \% & 20 \% \\ \text { Fairly good } & 45 \% & 5 \% \\ \text { Excellent } & 5 \% & 0 \%\end{array}$

Do you think your skin is smoother and softer?

\begin{tabular}{lll} 
Yes & $80 \%$ & $20 \%$ \\
No & $15 \%$ & $65 \%$ \\
No opinion & $5 \%$ & $15 \%$ \\
\hline
\end{tabular}


supplementation and continued over the entire study period. It is assumed that the ingested sphingolipids converted to sphingolipid metabolites which are absorbed through the intestine and distribute in the blood. Subsequently these metabolites reach the skin and play their physiological role to restore skin dryness [20].

In the present study a scoring scale for the diagnosis of the symptom severity was used to assess the efficacy of $A$. konjac extract. As a preliminary clinical trial this study included only the subjective measurement. Subjective scores are validated and clearly reflect the symptom severity. Even though the extent and intensity of skin condition are central to measure the severity, the symptoms of patients are subjective; hence an ideal scoring system should include the subjective symptoms [21,22]. The overall efficacy of $A$. konjac extract was found to be increasing significantly with time compared to the baseline. Our results agreed with the subject perception of the product efficacy as determined through the self-assessment questionnaire. Importantly, oral intake of $A$. konjac extract did not induce any toxic signs and was well tolerated throughout the study period. The present study, however, lacks the performance data collected via objective assessment.

\section{Conclusion}

In this clinical trial, we have shown that daily oral intake of A. konjac extract (glycosylceramides) significantly improved the skin health compared to placebo. However, considering the limitations of study being single-blinded and subjective, further trials are required using a double-blinded study design including the objective measurements to further explore the efficacy of $A$. konjac extract, and its effect on the enhancement of ceramide content in the skin.

\section{Supplementary information}

Supplementary information accompanies this paper at https://doi.org/10. 1186/s12906-019-2721-3.

\section{Additional file 1. Additional data. Characterization of SkinCera, Study event schedule, subject disposition, subject demographic data analysis, effect of SkinCera treatment on percentage subject response to skin parameters, analysis of vital signs and diagnosis scoring scale.}

Additional file 2. Questionnaire. Diagnosis questionnaire including the symptom scoring scale.

Additional file 3. Characterization of constituents in SkinCera. Details of other constituents of SkinCera apart from glycosylceramides 1-4.

Additional file 4. Sample size calculation.

\section{Abbreviations}

GCP: Good Clinical Practice; ICH: International Conference on Harmonization; LCMS/MS: Liquid chromatography-Mass Spectrometry

\section{Acknowledgements}

We sincerely express our gratitude for Syncorp Clincare Technologies (P) Ltd., India for their valuable support.

\section{Funds}

The present study is a part of research activities conducted by Vidya Herbs Pvt. Ltd., Bangalore, India. This clinical trial was funded by Vidya Herbs Pvt. Ltd. All the authors involved in the conceptualization, design and conduct of the study, and manuscript writing are employees of Vidya Herbs Pvt. Ltd.

\section{Author contributions}

SHV designed the study protocol and monitored the clinical trial. SHV also wrote the manuscript. NP performed the phytochemical analysis of the investigational product and assisted in manuscript preparation. SK conceived of the study and participated in its design and coordination and helped to draft the manuscript. All authors read and approved the final manuscript.

\section{Availability of data and materials}

The data sets used and/or analysed during the current study available from the corresponding author on reasonable request.

\section{Ethics approval and consent to participate}

This clinical trial was approved by the institutional ethics committee of Sri Kalabyraveshwara Swamy Ayurvedic Medical College, Hospital and Research Center, India. Subjects meeting all inclusion and no exclusion criteria signed a written informed consent and enrolled in the study.

\section{Consent for publication}

A statement that consent to publish is included in the manuscript. 'Consent to publish' is provided as supplementary file.

\section{Competing interests}

We have read and understood the BMC policy on declaration of interests and declare the following interests: All authors had financial support from the Vidya Herbs Pvt. Ltd. and declare the authors have a potential financial competing interest. The funding was oriented to institutional research support. The funding received from Vidya Herbs may in any way gain or lose financially from the publication of the article, either now or in the future.

Received: 27 August 2018 Accepted: 21 October 2019

Published online: 31 January 2020

\section{References}

1. Feingold KR, Elias PM. Role of lipids in the formation and maintenance of the cutaneous permeability barrier. Biochim Biophys Acta. 1841;2014:280-94

2. Jungersted JM, Hellgren LI, Jemec GB, Agner T. Lipids and skin barrier function-a clinical perspective. Contact Dermatitis. 2008;58:255-62.

3. Rabionet M, Gorgas K, Sandhoff R. Ceramide synthesis in the epidermis. Biochim Biophys Acta. 1841;2014:422-34.

4. Nilsson A, Duan RD. Alkaline sphingomyelinases and ceramidases of the gastrointestinal tract. Chem Phys Lipids. 1999;102:97-105.

5. Ueda O, Hasegawa M, Kitamura S. Distribution in skin of ceramide after oral administration to rats. Drug Metab Pharmacokinet. 2009;24:180-4.

6. Ueda O, Uchiyama T, Nakashima M. Distribution and metabolism of sphingosine in skin after oral administration to mice. Drug Metab Pharmacokinet. 2010;25:456-65.

7. Duan J, Sugawara T, Hirose M, Aida K, Sakai S, Fujii A, et al. Dietary sphingolipids improve skin barrier functions via the upregulation of ceramide synthases in the epidermis. Exp Dermatol. 2012;21(6):448-52.

8. Kajimoto $\mathrm{O}$. Clinical investigation of skin-beautifying effect of a beauty supplement containing rice-derived ceramide. J New Rem Clin. 2002;51:62-72.

9. Uchiyama T, Nakano Y, Ueda O, Mori H, Nakashima M, Noda A, et al. Oral intake of glucosylceramide improves relatively higher level of transepidermal water loss in mice and healthy human subjects. J Health Sci. 2008;54:559-66.

10. Hori M, Kishimoto S, Tezuka Y, Nishigori H, Nomoto K, Hamada U, et al. Double-blind study on effects of glucosyl ceramide in beet extract on skin elasticity and fibronectin production in human dermal fibroblasts. AntiAging Med. 2010;7:129-42.

11. Imokawa $\mathrm{G}$, Ishida K. Role of ceramide in the barrier function of the stratum corneum: implications for the pathogenesis of atopic dermatitis. J Clin Exp Dermatol Res. 2014;5:1.

12. Tessema EN, Gebre-Mariam T, Neubert RHH, Wohlrab J. Potential applications of phyto-derived ceramides in improving epidermal barrier function. Skin Pharmacol Physiol. 2017;30:115-38. 
13. Coderch L, De Pera M, Fonollosa J, De La Maza A, Parra J. Efficacy of stratum corneum lipid supplementation on human skin. Contact Dermatitis. 2002;47:139-46.

14. Usuki S, Tamura N, Sakai S, Tamura T, Mukai K, Igarashi Y.

Chemoenzymatically prepared konjac ceramide inhibits NGF-induced neurite outgrowth by a semaphorin 3A-like action. Biochem Biophys Rep. 2016;5:160-7.

15. Usuki S, Tamura N, Yuyama K, Tamura T, Mukai K, Igarashi Y. Konjac ceramide (KCer) regulates NGF-induced neutrite outgrowth via the Sema3A signaling pathway. J Oleo Sci. 2018;67(1):77-86.

16. Zhang J, Hou W, Feng S, Chen X, Wang H. Classification of facial wrinkles among Chinese women. J Biomed Res. 2017;31(2):108-15.

17. Beer KR. Combined treatment for skin rejuvenation and soft-tissue augmentation of the aging face. J Drugs Dermatol. 2011;10(2):125-32.

18. Ono J, Kinoshita M, Aida K, Tamura M, Ohnishi M. Effects of dietary glucosylceramide on dermatitis in atopic dermatitis model mice. Eur I Lipid Sci Technol. 2010;112:708-11.

19. Shimoda H, Terazawa S, Hitoe S, Tanaka J, Nakamura S, Matsuda H, et al. Changes in ceramides and glucosylceramides in mouse skin and human epidermal equivalents by rice-derived glucosylceramide. J Med Food. 2012; 15:1064-72.

20. Bizot V, Cestone E, Michelotti A, Nobile V. Improving skin hydration and age-related symptoms by oral administration of wheat glucosylceramides and digalactosyl diglycerides: a human clinical study. Cosmetics. 2017;4:37.

21. Luger T, Van Leent EJ, Graeber M, Hedgecock S, Thurston M, Kandra A, et al. An emerging safe and effective treatment for atopic dermatitis. $\mathrm{Br} J$ Dermatol. 2001;144:788-94.

22. Paller A, Eichenfield LF, Leung DY, Stewart D, Appell M. A 12-week study of tacrolimus ointment for the treatment of atopic dermatitis in pediatric patients. J Am Acad Dermatol. 2001;44:47-57.

\section{Publisher's Note}

Springer Nature remains neutral with regard to jurisdictional claims in published maps and institutional affiliations.

Ready to submit your research? Choose BMC and benefit from:

- fast, convenient online submission

- thorough peer review by experienced researchers in your field

- rapid publication on acceptance

- support for research data, including large and complex data types

- gold Open Access which fosters wider collaboration and increased citations

- maximum visibility for your research: over $100 \mathrm{M}$ website views per year

At $\mathrm{BMC}$, research is always in progress.

Learn more biomedcentral.com/submissions 